\title{
REMINISCENCIAS DE GEORG HENRIK VON WRIGHT *
}

\author{
Eugenio Bulygin \\ Universidad de Buenos Aires
}

RESUMEN. En este texto se describen algunos recuerdos y anécdotas de los encuentros del autor con

G. H. VON WRIGHT y de sus discusiones e intercambios sobre lógica y filosofía.

Palabras clave: G. H. von WRIGHT, E. BULYGIN, lógica de las normas.

\section{Reminiscences of Georg Henrik von Wright}

ABSTRACT. This is a collection of some of the memories and anecdotes that the author shares with G. H. VON WRIGHT. We can also read his discussions and exchanges on logic and philosophy.

Keywords: G. H. von WRIGHT, E. BuLYGIN, deontic logic.

* Fecha de recepción: 5 de noviembre de 2015. Fecha de aceptación: 1 de septiembre de 2016.

Traducción de Pablo E. NAVARRO. 
n día vi el anuncio de un nuevo seminario sobre lógica deóntica, a cargo del profesor Carlos Cossio, en el Instituto de Filosofía Jurídica de la Universidad de Buenos Aires. Fue a comienzo de la década del cincuenta, es decir, muy poco después de la publicación del primer artículo de VON WRIGHT sobre lógica deóntica. El apellido «VON WRIGHT» me impresionó como extraño, a causa de la combinación inusual del alemán «von» y el inglés «Wright». Asistí a dos o tres reuniones del seminario sin mucho beneficio. En esos tiempos era un estudiante de grado de la carrera de derecho y, aunque ya estaba interesado en la filosofía, estaba poco familiarizado con la idea de la lógica, y por ello, no pude seguir el artículo de VON WRIGHT, ni la discusión en el seminario. Pero conocí allí a un joven que desempeñó un papel destacado en mi vida: Carlos ALCHOURRÓN. Carlos, quien era de mi generación y también era estudiante de derecho, ya era un connoisseur de la lógica y la filosofía analítica. Dado que teníamos intereses similares comenzamos a trabajar juntos y nos convertimos en buenos amigos. Esta amistad duro más de cuarenta años y fue realmente fructífera ya que escribimos conjuntamente diversos libros y una buena cantidad de artículos.

En 1955 se produjo un cambio político importante en Argentina: el presidente Perón, cuyo gobierno fue (al menos en Educación) muy reaccionario, fue removido por una revolución militar y ello tuvo una profunda influencia en la Universidad. Durante el régimen de Perón, la filosofía del derecho estuvo dominada por personas formadas en la tradición del Derecho natural, con la excepción de Cossio. Desafortunadamente, él también perdió su posición académica en esa ocasión, pero su sucesor fue Ambrosio GIOJA, un respetado filósofo y un destacado profesor, así que ambos, Carlos y yo, comenzamos a colaborar activamente con él. GIOJA era un seguidor de KANT y HusSERL en el campo de la filosofía general y un gran admirador de la filosofía jurídica de KELSEN. Su idea era usar la fenomenología como la base filosófica para la Teoría Pura del Derecho de KELSEN y Carlos pretendía hacer los mismo, pero usando la lógica y la filosofía analítica. La confrontación entre esas dos personalidades tan diferentes, un profesor y un estudiante recién graduado (graduado con un promedio muy bajo) tuvo un resultado sorprendente: después de varios años de intensas discusiones fue Carlos quien ganó la batalla. El clima filosófico del Instituto cambió completamente. HusSERL y SCHELER fueron reemplazados por CARNAP, TARSKI, VON WRIGHT y WITTGENSTEIN y, en filosofía jurídica, junto con KELSEN aparecieron los trabajos de Alf Ross y H. L. A. HART, traducidos al castellano por otro gran amigo y excelente filósofo del derecho: Genaro CARRIÓ, quien en 1984, con la presidencia de Alfonsín, se convirtió en presidente de la Corte Suprema de Justicia de Argentina.

\section{(II)}

Como dije anteriormente, mi primer encuentro con los trabajos de VON WRIGHT fue un fracaso. Pero pocos años después leí su Norm and Action, que fue publicado en 1963. Este libro me impresionó mucho y decidí traducirlo al castellano, junto con otro 
de mis grandes amigos, Ernesto GARZÓN VALDÉs. Pero cometimos el error de no requerir inmediatamente la autorización del editor y, cuando lo hicimos, los derechos de la traducción ya habían sido vendidos a un editor español. Nuestra traducción nunca fue publicada, a pesar de ser considerablemente mejor que la publicada en Madrid ${ }^{1}$. Pero este fracaso nos dio la oportunidad de contactar con VON WRIGHT y de invitarlo a Argentina. Él estaba bastante entusiasmado con la invitación, pero entonces un desastre político ocurrió en Argentina: un coup d'état militar. El gobierno democrático fue reemplazado por una dictadura militar y todas las autoridades universitarias (rectores y decanos) fueron removidas y, en muchos casos, reemplazados por coroneles y capitanes ${ }^{2}$. Como resultado, no podíamos contar con la Universidad de Buenos Aires para financiar nuestra invitación. Afortunadamente, el nuevo rector de la Universidad de La Plata (Capital de la Provincia de Buenos Aires) no era un sargento sino un arquitecto y un viejo amigo de Ernesto, y se mostró de acuerdo con invitar a VON WRIGHT a Argentina. Esa fue la razón por la que VON WRIGHT no fue invitado por la principal universidad del país, sino por una universidad ubicada en la provincia de Buenos Aires.

En 1968, el mismo día de la llegada de vON Wright, Ambrosio Gioja, Ernesto GARZÓN, Carlos ALCHOURRÓN y yo estábamos sentados junto con VON WRIGHT en la recepción de su hotel, conversando sobre sus próximas clases. Ya sabíamos acerca de qué se trataban porque él nos había enviado un par de semanas antes el manuscrito de un nuevo libro, que utilizaría como base de sus conferencias. En ese libro, él distinguía entre seis conceptos de obligación y seis conceptos de permisión, que eran definidos en términos de mundos posibles a través de sus correspondientes fórmulas. Durante la conversación ALCHOURRÓN tímidamente expreso sus dudas acerca de esas fórmulas y en una servilleta de papel comenzó su demostración (su «truco sucio» como lo llamo VON WRIGHT). En cinco minutos VON WRIGHT se dio cuenta de que las fórmulas eran, en verdad, erróneas (ALCHOURRÓN probó que las seis definiciones eran equivalentes). Así que las eliminó del texto de su libro, que apareció en el mismo año ${ }^{3}$. VON WRIGHT quedó muy impresionando con la crítica de ALCHOURRÓN, pero lejos de sentirse contrariado, fue el principio de nuestra amistad.

Las conferencias de VON WRIGHT en La Plata, a 60 kilómetros de Buenos Aires, fueron muy populares, pero los asistentes fueron principalmente de la Ciudad de Buenos Aires. Fueron cuatro conferencias en las que desarrolló las principales ideas de su libro y fueron seguidas de una viva discusión. Más tarde dio dos conferencias en Buenos Aires, y fue posteriormente invitado por la Universidad de Córdoba (una ciudad colonial a 760 kilómetros de Buenos Aires). Carlos, Ernesto y yo le acompañamos a Córdoba, donde dio un par de conferencias. En esa ocasión, visitamos santa Catalina, un hermoso monasterio barroco, construido por los Jesuitas en el siglo XVII, en una

${ }^{1}$ La traducción publicada por Tecnos, una bien conocida y prestigiosa editorial, tiene muchos errores, especialmente en el capítulo III. La misma primera frase, en la p. 21, dice: «La palabra inglesa "norma”...». La palabra «norma», ciertamente, no es una palabra inglesa.

2 Cfr. E. BulYgin y E. GARZÓN VAldÉS, «Ursprung und Entwicklung der analytischen Rechtsphilosophie in Argentinien», en E. BulYgin y E. GaRZÓN VALDÉs (Hrg.), Argentinische Rechtsphilosophie heute, Berlin, Duncker \& Humblot, 1987, 7-11.

${ }^{3}$ G. H. von Wright, An Essay in Deontic Logic and the General Theory of Action, Amsterdam, North Holland, 1968. En su prefacio, vON WRIGHT reconoce la crítica de ALCHOURRÓN: «Agradezco al profesor Carlos ALCHOURRÓN por una importante corrección a mi tratamiento previo del cálculo deóntico diádico». 
«Estancia», que sirvió como la base financiera de la Universidad de Córdoba, una de las primeras universidades en Sudamérica. Luego de la expulsión de los Jesuitas por parte de la corona española (1767), fue comprada en un remate público por un antepasado de la familia de mi mujer. Algunos años después fue declarada Patrimonio de la Humanidad por la UNESCO, pero aún es propiedad de la familia y uno de nuestros lugares favoritos de vacaciones. VON WRIGHT quedó maravillado con el lugar y en sus otras visitas a Argentina pasó, junto con su esposa, bastantes días en el (antiguo) monasterio.

Los tres estuvimos en contacto con VON WRIGHT todo ese tiempo y cuando él dejo Argentina ya éramos (a pesar de las diferencias de edad y conocimiento) grandes amigos. Estoy seguro de que él fue muy feliz en este primer y más bien extenso viaje a Argentina ${ }^{4}$.

(III)

En el mismo año 1968 obtuve una beca del British Council, que me permitió pasar todo un año en Oxford. Mi supervisor fue H. L. A. HART. Durante mi estancia allí fui invitado por VON WRIGHT a visitarlo en Cambridge, donde tuve la oportunidad de discutir con él numerosos problemas del libro que estaba escribiendo con ALCHOURRÓN y de reunirme con algunos importantes filósofos y matemáticos: entre otros, C. D. BROAD, Casimir LEwy y John LitTLEwoOd. En Oxford asistí a muchos seminaries y clases, especialmente aquellas de Peter StraWsOn, Arthur PRIOR, Frederick AyER, John MACKIE y Anthony KenNY, a las que seguí asiduamente durante los tres trimestres académicos. Pero mi principal preocupación era el libro que estaba escribiendo con ALCHOURRÓn y tuve numerosas y esclarecedoras discusiones con PRIOR, MACKIE y, por supuesto con H. L. A. HART. Recuerdo especialmente a una de ellas acerca de los permisos y las normas permisivas. En principio, HART, al igual que muchos otros filósofos del derecho, como, por ejemplo, Alf Ross, era más bien escéptico acerca de las normas permisivas, pero luego de varias reuniones cambió de opinión.

Las discusiones con HART, VON WRIGHT y PRIOR contribuyeron mucho a la elaboración de nuestro libro, que terminamos en 1970. El problema de dónde publicarlo fue resuelto gracias a Mario BUNGE, que nos ofreció su nueva colección llamada Library of Exact Philosophy, publicada por Springer (Viena y Nueva York). El paso siguiente fue traducirlo al inglés, lo que fue realizado gracias a la invalorable asistencia de Joan, la esposa de John MACKIE, y así el libro apareció publicado por Springer. En 1975 apareció la versión original en castellano con el más bien oscuro título de Introducción a la Metodología de las Ciencias Jurídicas y Sociales (Astrea, Buenos Aires), que, más tarde, en una nueva edición del 2012, fue cambiado a Sistemas Normativos. Durante los años subsiguientes el libro fue traducido al alemán (1994), italiano (2005), ruso (editado dos veces en 2010 y 2013) y portugués. A pesar de sus años, es aún leído y comentado en muchos países.

${ }^{4}$ Cfr. Su autobiografía MITT LIV SOM JAG Minns det, Albert Bonniers Förlag, 2001, 264-269. 
En diciembre de 1971 fui invitado a Finlandia por el rector de la Universidad de Helsinki (conforme a una propuesta de VON WRIGHT). Llegue en medio de una tormenta de nieve y me sorprendió verlo allí, en el aeropuerto, esperándome. Mi visita a Finlandia fue muy estimulante. Di una conferencia sobre la «Norma Básica» en la Universidad de Helsinki y me reuní en diferentes ocasiones con algunos importantes filósofos como, por ejemplo, Jaakko HinTIKKA, Eric STENIUS y Aulis AARNiO. Pero lo más importante para mí fue encontrarme con la familia de VON WRIGHT: su esposa Elisabeth y su hijo Benedict. Más tarde tuve ocasión de conocer al resto: su hija Anita y su esposo Tom GRÖNBERG, a sus hijas Johanna y Helena, al igual que a la mujer de Benedict, Tytii, y su hija Naivasha.

En 1975 asistí a un coloquio sobre «Lógica deóntica y semántica», en la Universidad de Bielefeld, Alemania, que fue organizado por vON WRIGHT y Amedeo CONTE. Presenté un artículo titulado «Unvollständigkeit, Widersprüchlichkeit und Unbestimmtheit der Normenordnungen» ${ }^{5}$. Aunque el trabajo fue escrito por mí, el mismo era el resultado de una investigación conjunta con Carlos acerca del concepto de derogación, un concepto ampliamente conocido por los juristas y abogados, pero olvidado por los filósofos del derecho y los lógicos deónticos. VON WRIGHT consideró a este trabajo como «la más significativa y original contribución de ALCHOURRÓN y BULYGIN a la teoría de las normas» ${ }^{6}$ y también añadió que en esa ocasión, él se dio cuenta por primera vez que el tratamiento lógico del problema de la derogación mostraba que la lógica deóntica podría resultar útil no solo para los lógicos sino también para la teoría jurídica ${ }^{7}$.

En 1982, Carlos y yo asistimos al Congreso Mundial de la IVR, realizado en Helsinki ${ }^{8}$. Fue una notable reunión: VON WRIGHT dictó la conferencia inaugural sobre «Ser y deber ser» y Aulis Aarnio fue elegido presidente de la IVR. Durante el congreso, me hospedé en el apartamento de Georg Henrik en Helsinki y tras del congreso, él y Elizabeth me invitaron a su villa en Valö, ubicada en un hermoso lugar de una pequeña isla del archipiélago de Finlandia. Disfruté mucho los días que pasé en ese tranquilo lugar: excursiones en lancha por el archipiélago, caminatas por los bosques cercanos, recolección de setas (bajo la estricta supervisión de Elizabeth, quien impiadosamente rechazó algunos de mis más hermosos hallazgos, declarándolos venenosos) y discusiones con Georg Henrik. En los años siguientes estuve muchas veces en Valö, disfrutando de la hospitalidad de Elizabeth y Georg Henrik, al igual que de los hermosos entornos.

5 «Incompletitud, inconsistencia e indeterminación de sistemas normativos». Este artículo fue publicado en alemán en A. Conte, R. HilPINEn y G. Henrik vON Wright (eds.), Deontische Logik und Semantik, Wiesbaden, Athenaion, 1977, y en italiano por G. DI BERNARDO, Logica deontica e semantica, Bologna, 1977.

6 G. H. vON Wright, «Epilogue to the Festschrift for Alchourrón and Bulygin» en E. GARZÓN VALDÉs, W. Krawietz, G. Henrik von Wright y R. Zimmerling (eds.), Normative Systems in Moral and Legal Theory, Berlin, Duncker \& Humblot, 1997, 509-512.

7 Vid. Doxa núm. 1, 1984, 265-267.

8 International Association for Legal and Social Philosophy. 
En los años posteriores nos encontramos en muchas ocasiones en diferentes lugares: Roma, Venecia, Florencia, Palermo, Helsinki (o más bien, Helsingfors, como él llamaba a su ciudad, porque ellos pertenecían a la minoría lingüística sueca), Cambridge, Münster, Bonn, Bielefeld. Pero también en Argentina; ya que Georg Henrik y Elizabeth visitaron dos veces este país. Especialmente importante fue la visita en 1987, cuando viajaron a Buenos aires, Salta y Córdoba. En esa ocasión, VON WRIGHT recibió el grado de doctor Honoris Causa en las Universidades de Buenos aires y Salta y participó en el Congreso Internacional extraordinario de filosofía en Córdoba (organizado por FISP) ${ }^{9}$, donde dio una conferencia memorable en castellano, sobre Ciencia y Razón, recibiendo una ovación por parte principalmente de los estudiantes ${ }^{10}$. Este congreso reunió a un buen número de filósofos de diferentes países, como por ejemplo, Robert Alexy, Donald Davidson, Dagfinn FöllesDahl, Stig Kanger, Francisco Miró QueSADA, Ernesto SOSA, y el destacado lógico brasileño Newton DA COSTA, entre otros. Tuvimos un encuentro muy estimulante con Georg Henrik y Newton DA CosTA, discutiendo sobre las T-lógicas de VON WRIGHT.

Durante los años ochenta nos encontramos en los tres congresos de «Lógica, Informática, Derecho», organizados por Antonio MARTINO, director del Instituto para la Informática en Florencia. Recuerdo especialmente un largo viaje de Florencia a Palermo, tras del segundo congreso (1985). Georg Henrik, Carlos, Elizabeth y yo fuimos en coche, deteniéndonos en muchos lugares, visitando ciudades, iglesias y monasterios (como, por ejemplo, Sant'Antimo, cerca de Montalcino, un edificio románico del siglo XII maravilloso, con unas paredes de transparente alabastro) y nos quedamos tres días en la hermosa y pequeña ciudad de Ravello. En Palermo asistimos a la reunión anual del Institut International de Philosophie. Carlos y yo habíamos sido elegidos recientemente como miembros del Instituto, conforme a una propuesta de VON WRIGHT. Numerosos participantes presentaron sus trabajos y fueron intensamente discutidos. No solo los trabajos de QuiNE y vON WRIGHT, sino también los de Max BLACK, Jaakko HintikKa, Ruth BARCAN MARCUS y Francisco Miró Quesada fueron muy atractivos.

Las discusiones con Georg Henrik fueron mucho más importantes que las conferencias en los congresos y las visitas a diferentes lugares. En esas ocasiones, cada noche nos sentábamos en algún bar o pequeño café y Georg Henrik, bebiendo a sorbos su Fernet Branca, analizaba algún problema filosófico. Lo hacía de una manera especial. Escuchaba atentamente lo que otros decían acerca de un cierto tópico y luego replicaba en voz baja, dando sus argumentos. Mi memoria (o, mejor, mi falta de ella) no me permite reproducir los diálogos, pero afortunadamente entre muchas cartas de VON WRIGHT (tengo dos grandes carpetas repletas de ellas) hay algunas sobre problemas filosóficos. Casi todas nuestras cartas están escritas en alemán, pero hay algunas que Georg Henrik escribió en inglés para que Carlos ALCHOURRÓN pudiera leerlas y parti-

\footnotetext{
9 International Federation of Philosophical Societies.

10 Antes de la conferencia, pasamos varios días en Santa Catalina, donde G. Henrik pudo practicar la versión en castellano de su trabajo.
} 
cipase en la discusión. Haré algunas citas más bien extensas de esas cartas para ilustrar nuestras discusiones.

En una carta fechada en octubre de 1979, Georg Henrik escribe:

«Lieber Eugenio [...] Ich habe in den letzten Wochen viel über Norm- und Handlungslogik weitergedacht, im Anschluss an unseren Diskussionen in Argentina. Einige Seiten im Aufsatz für die Hilpinen-Anthologie habe ich korrigiert. Ich schicke Dir hier Kopien. (Meistens bandelt es sich um Unbedeutendes.) Weil das Folgende vielleicht auch für Carlos von Interesse sein kann, so schreibe ich den Rest des Briefes auf English» ${ }^{11}$.

Comentarios sobre algunos puntos de nuestras recientes discusiones:

1. La definición del predicado «permitido». Lo he definido de manera tal que una acción individual está permitida solo si no cae en ninguna categoría de acciones prohibidas y se encuentra en alguna categoría de acciones permitidas. ¿Por qué no decir simplemente que está permitida si y solo si cae en una categoría permitida? Entonces podría ocurrir que una misma acción individual fuese tanto permitida como prohibida — al igual que, en mi opinión, una misma acción individual puede ser obligatoria y prohibida (el caso de Jefté)—. Pero, me parece mejor no cambiar la definición, por la siguiente razón: si una acción individual es a la vez obligatoria y prohibida, entonces es un caso claro de conflicto («conflicto de obligaciones»). Si la acción, a su vez, cae bajo una clase prohibida y permitida, entonces me parece más natural decir que su carácter prohibido prevalece sobre la permisión. Sin embargo, la decisión parece ser principalmente una «cuestión de gustos». Sobre la cuestión importante estamos de acuerdo, pienso, es decir en que una norma que permite una clase de acción y otra norma que la prohíbe no hay un «contradicción lógica».

2. La cuestión de las normas derivadas (entailed norms). He estado pensando bastante sobre esto luego de nuestra discusión en casa de Eugenio y aún mantengo mi posición original. Ello significa que niego la validez lógica del principio de distribución para las obligaciones $\mathrm{O}(\mathrm{A} \& \mathrm{~B}) \rightarrow \mathrm{OA} \& \mathrm{OB}$ y la tesis $\mathrm{P}(\mathrm{A} \& \mathrm{~B}) \rightarrow \mathrm{PA}$. Si a alguien se le permite entrar a un jardín a través de una cierta puerta, no se le permite entrar al jardín simpliciter. Uno tiene el permiso solamente secundum quid, es decir, bajo la condición de (o dado que) uno ingrese a través de la puerta.

Sin embargo, dado que cualquier caso de entrar al jardín - en general, dado que (x) $([\mathrm{A} \& \mathrm{~B}] \mathrm{x} \rightarrow[\mathrm{A}] \mathrm{x}$ - se sigue por mi principio (5) que entrar al jardín no puede ser prohibido y por tanto tiene que ser permitido en sentido débil. Pero no creo que eso sea una «verdad lógica» sino un requisito de la «legislación racional».

De manera similar, se sigue de mi principio (6) que si las acciones de la clase A\&B son obligatorias, entonces las acciones de la clase A (y de la clase B) no pueden ser prohibidas.

Para decirlo de una manera más específica: las relaciones de implicación lógica entre normas son ilusorias. Acerca de esto, parece que ha existido un desacuerdo real entre nosotros - pero espero que podamos llegar a un acuerdo- .

3. También espero que podamos estar de acuerdo en que «hacer algo por hacer algo (más)», por ejemplo entrar a un jardín saltando una verja no es asimilable a los modificadores adverbiales como hacer algo lentamente o de manera reluctante o hacerlo bien. La acción individual de entrar al jardín saltando la verja cae bajo dos acciones genéricas, es decir, es un caso de entrar al jardín y un caso de saltar la verja. Es bastante diferente a, por ejemplo, conducir cuidadosamente. No es hacer dos cosas. No hay una acción genérica

11 «Querido Eugenio [...] en las últimas semanas he estado pensando bastante acerca de la lógica de normas y las acciones, en conexión con nuestra discusión en Argentina. Algunas páginas de mi paper para la antología de HILPINEN han sido corregidas. Les envío copias (principalmente no son muy significativas). Dado que lo siguiente puede ser de interés de Carlos, escribiré el resto de la carta en inglés». Aquí y en la siguiente discusión todos nos referimos al trabajo de VON WRIGHT, «On the Logic of Norms and Actions», publicado posteriormente en R. Hilpinen (ed.), New Studies in Deontic Logic, Dordrecht, Boston, London, Reidel, 1981, 3-35. 
«actuar cuidadosamente» - pero una acción genérica tal como conducir puede, en un caso individual, ser ejecutada cuidadosamente-.

Una cuestión importante por sí misma es cómo dar cuenta de enunciados de acción que contienen modificadores adverbiales. El problema ha sido bastante discutido en los años recientes - por Kenny, DavidSon, Toumela y otros-. Pero no estoy al tanto de alguna discusión del problema en contextos normativos (el ejemplo de Eugenio de estar permitido el conducir cuidadosamente). El problema podría ser interesante.

4. Un problema que me continúa preocupando concierne a la definición de las acciones individuales obligatorias. Sin embargo, el ejemplo de Orayen, en la última discusión en SADAF, no era, después de todo un buen ejemplo (si es que lo recuerdo correctamente). Un conductor llega a una intersección y ha de girar hacia la derecho o la izquierda. Él es consciente de la obligación y gira a la derecha. ¿Fue obligatorio eso que hizo? La acción de girar a la derecha no es obligatoria, pero la acción de girar hacia la derecho o la izquierda es obligatoria y, al girar a la derecha y no girar a la izquierda, el conductor ejecutó una acción individual que (también) cae bajo el carácter obligatorio de «girar a la derecha o la izquierda». Aqui no veo dificultad alguna.

Pero estoy preocupado por el caso en que la acción también constituye la omisión de algo. El caso del individuo que entra al jardín a través de la puerta y no salta la verja que tiene prohibido saltar. ¿Fue su acción obligatoria? Uno desearía decir NO. Lo obligatorio era su omisión de saltar la verja. Hasta aquí está todo bien. Pero si se puede decir de su acción que ella carecía de la propiedad de ser un salto de la verja y si la carencia de una propiedad prohibida convierte a la acción en obligatoria, entonces su acción entrar por la puerta fue obligatoria. Pero claramente no fue obligatoria - a pesar de que en un sentido fue «conforme al deber»-. Algo va mal aquí.

Si una acción individual tiene una propiedad que es tal que si la careciese sería entonces una acción prohibida, entonces la acción fue obligatoria. Esto, ciertamente, está bien. Pero si una acción individual carece de una propiedad que es tal que, si la hubiese tenido, hubiese sido una acción prohibida, entonces la acción no es (no es por eso convertida en) obligatoria. ¿Por qué esta asimetría? El problema es cómo la carencia de una propiedad de la acción se relación con el hecho de constituir una omisión.

Cualquier comentario tuyo o de Carlos me interesaría enormemente. Solo puedo esperar que no sean tan devastadores que tenga que retirar la publicación de mi trabajo de la antología de HiLPINEN.

$$
* * *
$$

La carta de Georg Henrik llega hasta aquí. Carlos y yo enviamos nuestros comentarios, pero desafortunadamente la respuesta de ALCHOURRÓn se ha perdido, o, al menos, he sido incapaz de encontrarla. Por ello, solo transcribiré mi réplica a vON WRIGHT.

Comentarios sobre los comentarios a nuestras discusiones:

I. Para clarificar el tema y seleccionar los problemas sobre los que estamos en desacuerdo sería aconsejable hacer algunas distinciones preliminares:

1. Hay tres tipos de enunciados normativos (normative sentences):

a) Enunciados normativos que expresan normas (formulaciones normativas, p. 48) ${ }^{12}$ : para evitar ambigüedades (que tú pareces ansioso por mantener), usaré los símbolos !A y ! A para ordenes y prohibiciones, respectivamente. Para normas permisivas propongo usar *A.

12 Aparentemente esos números de páginas corresponden al artículo de VON WRIGHT, pero no coinciden con la versión impresa. 
b) Enunciados normativos que expresan proposiciones normativas: OA, FA, PA.

c) Enunciados normativos que expresan predicaciones deónticas: $\mathrm{Ox}, \mathrm{Fx}, \mathrm{Px}$.

2. Estamos de acuerdo [aunque tú pareces no estar consciente de ello —cfr. tu énfasis (punto 2 de tu carta) acerca de las normas derivadas-] en que no hay lógica de normas, es decir, no hay relaciones lógica entre expresiones del tipo !A; por ello no hay normas implicadas o derivadas (o más bien, al revés: porque no hay normas derives es que no hay lógica de normas).

Sobre este punto hay un cambio significativo en nuestras posiciones (tal como manifestamos en nuestro artículo para el volumen de SCHILPP). Está explicada en detalle en «La concepción expresiva de las normas» y tú no la has objetado. Así que aquí todo es pax et concordia.

3. Hay una lógica de proposiciones normativas o de operadores deónticos como $\mathrm{O}, \mathrm{F}$ y $\mathrm{P}$ (por brevedad, LDO). Claramente la verdad de las proposiciones normativas depende de la existencia de normas. Si hay una norma!A, entonces OA es verdadera (la conversa no necesariamente se mantiene: OA podría ser verdadera aun si no hay tal norma como !A —en el sentido de haber sido prescripta por una autoridad-. Sobre este punto, pienso, también hay acuerdo).

4. Hay también una lógica de la predicación deóntica (LDP), pero tal lógica (a) sería diferente de LDO y (b) dependiente de LDO (como tú dices: «...tenemos que considerar los predicados deónticos como secundarios respecto a los operadores deónticos». P. 48), pero no viceversa: $\mathrm{LDO}$ es independiente de LPD.

Este punto es muy importante: lo que dices en la p. 48 parece confirmarlo, pero como veremos ahora, no siempre te mantienes consecuente con esa idea.

II. En el capítulo III tartas de desarrollar una LDO; nosotros hicimos prácticamente lo mismo en nuestro trabajo sobre la Concepción Expresiva. Pero los resultados son diferentes. Aquí debe haber algún desacuerdo sustancias. ¿Cuál es?

1. No trataré de elucidar tal desacuerdo (si es que hay alguno). Tal vez Carlos lo haga (por cierto, estamos escribiendo de manera independiente, así que puede haber algún solapamiento). Lo que haré, por el contrario, es destacar lo que considero un error metodológico general en tu argumento. Si ese error es admitido, entonces, tal vez, no haya desacuerdo en absoluto, o al menos, se podrá ver con más claridad. Si no hay tal error (lo que es muy probable), entonces todo lo que sigue es un puro sin sentido.

2. Tú aceptas el Principio de Equivalencia Deóntica (p. 49), pero rechazas el principio más fuerte (que nosotros aceptamos) de la Obligación Derivada, según el cual las consecuencias lógicas de acciones (genéricas) obligatorias son obligatorias. (A pesar de que no hay normas derivadas, hay proposiciones normativas derivadas o implicadas).

En particular, rechazas las fórmulas $\mathrm{P}(\mathrm{A} \& \mathrm{~B}) \rightarrow \mathrm{PA}$ y $\mathrm{O}(\mathrm{A} \& \mathrm{~B}) \rightarrow \mathrm{OA} \& \mathrm{OB}$ (p. 53, punto 2 de tu carta).

Veamos el argumento que utilizas para ese rechazo:

El hecho de que una acción individual con las dos características sea permitida no es una garantía de que una acción individual que solo tiene una de ellas sea permitida (p. 53).

Esto es perfectamente verdadero, pero irrelevante para la discusión. Nosotros no estamos interesados en acciones individuales sino con acciones genéricas; lo que la fórmula dice es: si la acción genérica $A \& B$ está permitida, entonces la acción genérica $A$ está permitida. Esto no significa que todas las acciones individuales que caen bajo la categoría A también están permitidas (cfr. p. 51: «Del hecho de que las acciones de un cierto tipo sean permitidas no se sigue que todas las acciones individuales de ese tipo sean acciones permitidas»).

Supóngase que hay una norma que permite A (*A); por tanto PA es verdadero. ¿Significa esto que todas las acciones individuales con la característica A están permitidas? Ciertamente no. La normas que permite entrar al jardín simpliciter, puede coexistir con la norma que prohíbe saltar verjas, simpliciter. En el caso de la acción individual de entrar al 
jardín saltando una verja se trata de una acción individual tanto permitida como prohibida y de allí, conforme a tu definición del predicado «permitido», está prohibida. Pero si la permisión de una acción genérica es compatible con la prohibición de una acción individual del correspondiente tipo, entonces tu argumento de la p. 53 es irrelevante.

3. El mismo argumento es aplicable a la fórmula $\mathrm{O}(\mathrm{A} \& \mathrm{~B}) \rightarrow \mathrm{OA} \& \mathrm{OB}$. Aquí, nuevamente, el recurso a las acciones individuales es irrelevante. Si uno tiene una obligación de entrar al jardín a través de una cierta puerta, entonces uno también tiene una obligación de entrar al jardín simpliciter. Pero la obligación de entrar al jardín no excluye la posibilidad de que la acción individual de entrar al jardín pueda ser tanto obligatoria como prohibida. Esto depende de otras normas y por ello también de la obligatoriedad o prohibición de otras acciones genéricas.

4. En general, no se puede decidir si una acción individual de una cierta clase es permitida, prohibida u obligatoria sobre la base únicamente de las calificaciones deónticas de una cierta clase (acciones genéricas). De allí que tu argumento en favor de los principios lógicos de LDO sobre la base de consideraciones referidas a las acciones individuales es radicalmente equivocado. Desafortunadamente, esta clase de errores se repite frecuentemente (e.g., pp. 50-57).

5. En p. 57 dices:

Los hechos sobre los que he basado mis argumentos para aceptar y para no aceptar ciertos enunciados acerca de la relación entre normas (¿no sería mejor hablar de proposiciones normativas?) son verdades lógicas. Ellos son derivados de la definición de predicados deónticos con la ayuda de los principios de la lógica de la predicación.

Esto es exactamente lo que me parece objetable. Los principios de LDO son independientes de LDP y por ello no pueden ser derivados de las definiciones de DP y LP.

Mi hipótesis es ahora que nuestros desacuerdos acerca del principio de LDO se deben principalmente al modo equivocado que empleas para justificarlos. Si logramos acuerdo en este punto, entonces tal vez no hay desacuerdo en absoluto, o al menos, considerablemente menos desacuerdos sobre los principios de LDO.

III. Ahora comentaré directamente los temas que surgen en tu carta:

Ad1. Todo el problema de las acciones individuales y sus predicados deónticos, para mí, está bastante poco claro. Ni siquiera estoy seguro de que hay algo así como LDP y cuáles deberían ser sus principios. Pero hay un punto importante del que no fui consciente durante nuestra discusión, viz. Que tu definición de «permitido» se aplica solo a las acciones individuales. Estaré perfectamente feliz si admites - como parece hacer-que puede haber normas que permiten y prohíben la misma acción genérica. Pero aún así, no estoy muy feliz con tu definición del predicado «permitido». Tal vez haya que distinguir dos casos:

Caso 1: hay una norma permitiendo A y una norma permitiendo B, así PA y FB son verdaderas. Una acción individual $[\mathrm{A} \& \mathrm{~B}] \mathrm{x}$ sería «naturalmente» considerada como prohibida.

Caso 2: hay una norma permitiendo A y una norma prohibiendo A; así PA y FA son verdaderas. ¿Qué diríamos de la acción individual [A]x? Esto, pienso, dependería de cómo resolvamos el conflicto entre ambas normas. Si la norma que permite A ha sido promulgada por una autoridad superior, entonces $[\mathrm{A}] \mathrm{x}$ sería considerada como permitida, a pesar de la otra norma. Así que no parece haber un método directo para decidir el caso: primero debe resolverse el conflicto de normas (mediante derogación o imponiendo un orden). Pero, en tanto que el conflicto no es resuelto, la acción individual es tanto permitida como prohibida.

$* * *$

Georg Henrik no aceptó mis críticas, pero expresó algunas dudas sobre sus ideas de construir la lógica deóntica sobre la base de la predicación deóntica, i. e. sobre enunciados que señalan que las acciones individuales tienen la propiedad de ser obligatorias, prohibidas o permitidas. El trasfondo de nuestras discusiones fueron los dos 
artículos ya mencionados: su «On the Logic of Norm and Action» y nuestro «The Expressive Conception of Norms». Ambos trabajos fueron posteriormente publicados en la antología de Hilpinen, pero ya eran conocidos por los tres participantes de la discusión.

Como he enfatizado en la carta que he transcripto, en la nueva concepción de VON WRIGHT sobre la lógica deóntica aparecen tras clases de expresiones normativas: a) formulaciones normativas que expresan normas, $i$. e., órdenes, prohibiciones y permisos; $b$ ) proposiciones normativas que establecen que ciertas acciones genéricas son obligatorias, prohibidas o permitidas, y $c$ ) predicaciones normativas, que establecen que una acción individual es obligatoria, prohibida o permitida. Mientras que en «The Expressive Conception» hay solo dos clases de expresiones normativas: normas y proposiciones normativas. Las normas son concebidas como prescripciones de acciones genéricas como obligatorias, prohibidas o permitidas. Ciertamente no ignorábamos la diferencia entre acciones individuales y genéricas (acerca de la distinción entre casos individuales y genéricos y soluciones individuales y genéricas, véase Normative Systems, pp. 35 y 153-154). Pero dado que los caracteres deónticos de las acciones individuales dependen exclusivamente de las calificaciones de las acciones genéricas nos parecía que la predicación deóntica de las acciones individuales carece de interés autónomo.

Esos dos diferentes enfoques conducen a algunos problemas. VON WRIGHT admite que una acción individual puede ser obligatoria y permitida si cae bajo dos acciones genéricas, una de la cual es obligatoria y la otra es prohibida. Llama a ello «conflicto de obligaciones». Pero si una acción individual cae bajo la prohibición y la permisión de una acción genérica, entonces por su definición de permisión, prevalece el carácter prohibido sobre la permisión. ¿Por qué? Esas situaciones han sido analizadas en «The Expressive Conception» bajo el título de conflictos de ambivalencia y aun no veo por qué una prohibición debe prevalecer sobre la permisión ${ }^{13}$.

En abril de 1996 tuvo lugar un coloquio Internacional en la Universidad de Bielefeld, organizado por Georg MEGGLE, quien también editó el correspondiente volumen ${ }^{14}$. En el artículo de VON WRiGHT, «Value, Norm, and Action in my Philosophical Writings», encontramos la siguiente afirmación:

Los primeros en ver claramente la necesidad de distinguir entre una lógica de proposiciones normativas y una lógica de normas (una lógica deóntica «real»), pienso, fuero Carlos AlChOURRÓN y Eugenio BulYGIN, co-autores del clásico Normative Systems. Unos pocos años antes de la publicación del libro, ALCHOURRÓN hizo su primer intento de construir una lógica de proposiciones normativas —o como él la llamaba una «lógica normativa»- en contraposición a la lógica de normas. Es un gran mérito de los autores haber comprendido esta doble tarea del estudio lógico de las normas. He tardado casi treinta años en ver su completa significación. Y no todos los lógicos y filósofos la han comprendido ni tan siquiera ahora.

Sería un tanto presuntuoso conjeturar que nuestras discusiones de finales de 1979 y 1980 — que claramente caen en el período de los treinta años mencionados por vON

\footnotetext{
13 «The Expressive Conception of Norms», 106-108.

14 G. Meggle (ed.), Actions. Norms, Values, Discussions with Georg Henrik von Wright, Berlin, De Gruyter, 1999.
} 
WRIGHT- podría haber influenciado el cambio en su concepción de la lógica deóntica. Pero es innegable que hubo un cambio en el sentido que desde la publicación de su artículo de 1981, nunca retomo la idea de la predicación deóntica. En su extenso e importante artículo «Norms, Truth and Logic» ${ }^{15}$, la lógica deóntica se basa en principios de una legislación racional. Tales principios fueron vistos en primer término como «causi-lógicos», pero finalmente VON WRIGHT acepto que las leyes basadas en la noción de ejecutabilidad (doability) eran leyes lógicas. Este punto es claramente reafirmado en su artículo «¿Hay una lógica de normas?» ${ }^{16}$ y en el trabajo publicado en el volumen de MEGGLE. Las nociones de consistencia y contradicción entre normas son allí analizadas sobre la base de consideraciones semánticas ${ }^{17}$.

(VI)

No solo discutíamos filosofía. Algunas veces también conversábamos sobre literatura. Georg Henrik hablaba con fluidez numerosos idiomas: sueco, alemán, inglés y finlandés. También tenía un buen conocimiento de francés, italiano y ruso. Su conocimiento de literatura era sorprendente. Sus favoritos, dejando aparte a GOETHE y SCHILLER, eran Thomas MANN y MusiL. En una carta que me envió en los setenta me decía que había comenzado a aprender ruso para ser capaz de leer a los autores rusos. Para mi sorpresa, en un par de años ya estaba leyendo a TOLSTOY, DOSTOYEVSKY y BULGAKOV, cuyo El Maestro y Margarita, era para él una de las grandes novelas del siglo veinte. Georg Henrik publicó en sueco dos ensayos sobre TOLSTOY y DOSTOYEvSKY, que fueron luego traducidos al inglés y al ruso ${ }^{18}$. Ambos ensayos son un ejemplo de lo mejor que se ha escrito acerca de las ideas filosóficas y religiosas de esos grandes pensadores.

En algún momento (no recuerdo exactamente cuándo), en alguna reunión filosófica (no recuerdo dónde), alguien (no recuerdo quién) me dio un paquete que enviaba Georg Henrik: contenía una copia de su último libro Six Essays in Philosophical Logic, con una dedicatoria:

\author{
To \\ Carlos Alchourrón (†) \\ and \\ Eugenio Bulygin \\ friends and companions \\ in an intellectual adventure*
}

Esta ha sido, con mucha diferencia, la más importante distinción que he recibido en mi vida académica. El único punto lamentable de ella fue el hecho de que Carlos (quien murió en 1996) no llegase a verla.

15 En G. H. von Wright, Practical Reason, vol. I of Philosophical Papers, Oxford, Basil Blackwell, 1983.

16 G. Henrik von Wright, Six Essays in Philosophical Logic, Acta Philosophica Fennica, vol. 60, 1966.

17 «Value, Norm, and Action in My Philosophical Writings», 21.

18 G. H. VON WRight, Tanke och förkunnelse, 1955, Tрu мыслителя (Three thinkers), Saint Petersburg, 2000.

* Nota del traductor: A Carlos Alchourrón ( $\dagger$ y Eugenio Bulygin. Amigos y compañeros en una aventura intelectual. 


\section{(VII)}

Georg Henrik VON WRIGHT murió en Helsinki el 16 de Junio del 2003, a la edad de 87 años. Su muerte puso punto final a nuestras cartas, conversaciones y discusiones, pero no al estudio de su obra ni al contacto telefónico, electrónico o personal con Elizabeth y el resto de la familia.

En 2004 tuve el privilegio de participar en el simposio In Memoriam Georg Henrik von Wright, realizado en el Aula Magna de la Universidad de Helsinki y organizado por la Sociedad Filosófica de Finlandia, de la cual fue presidente durante muchos años. 11 contribuciones de reconocidos filósofos y amigos de VON WRIGHT fueron publicados en esa ocasión para un volumen especial ${ }^{19}$.

Antes del simposio, fuimos con Elizabeth y Anita a Valö. Fue una experiencia más bien triste, debido a la ausencia de Georg Henrik. Cada detalle del lugar recordaba a Georg Henrik, pero él ya no estaba allí.

En 2007 fui invitado a la celebración del 90 cumpleaños de Elizabeth, en la hermosa casa de Anita, en Ollas, cerca de Helsinki. Elizabeth estaba, como siempre, espléndida, alegre e ingeniosa, e incluso bailó en aquella ocasión. Estuve con ellos tres días maravillosos: sentí que era un miembro de la familia y aun me considero como tal. Espero que esa no sea mi última visita a Finlandia, un país al que aprecio enormemente.

Poco tiempo después, Elizabeth también dejó este mundo. Fue una mujer maravillosa: una belleza célebre en su juventud y me inclino a sostener que era aún más bella cuando la conocí en la década de los setenta y que conservó su encanto hasta el final. Excepcionalmente inteligente e ingeniosa, ella fue una compañera perfecta para Georg Henrik.

\section{(VIII)}

Georg Henrik vON WRIGHT fue un gran lógico y filósofo. Puede ser considerado como uno de los fundadores de la lógica filosófica ${ }^{20}$. Sus escritos sobre lógica deóntica, al igual que sobre la lógica del cambio, la acción, el tiempo, la preferencia y, finalmente aunque no por ello menos importante, sobre la lógica de la verdad (T-lógicas) son de fundamental importancia. Él también escribió libros y artículos sobre muchos otros problemas filosóficos, tales como la inducción, probabilidad, libertad y determinismo, valores, y en todos esos tópicos hizo contribuciones perdurables.

Sin embargo, VON WRIGHT no escribió solo libros y artículos para filósofos. Estaba profundamente preocupado por cuestiones concernientes a nuestra situación en el mundo, por las condiciones políticas, económicas y sociales de nuestra vida. Publicó artículos en periódicos y revistas, principalmente en sueco, sobre tópicos tales como el

19 I. NiINILUOTO y R. ViLKKO (eds.), Philosophical Essays in Memoriam Georg Henrik von Wright, Acta Philosophica Fennica, vol. 77, 2005.

20 J. HintikKA, «G. H. von Wright on Logic, Philosophy and Mathematics», en I. Ninniluoto y R. VILKKO (eds.), Philosophical Essays in Memoriam Georg Henrik von Wright, Helsinki, 2005, 33-43. 
consumismo materialista que conduce al agotamiento de los recursos naturales, la carga del medio ambiente por acumulación de residuos, los peligros de la energía atómica, las guerras, la tortura y esos artículos fueron muy influyentes no solo en Finlandia y los otros países escandinavos. Algunos de esos trabajos han sido recogidos en sus libros ${ }^{21}$.

No trataré de dar una idea de sus logros filosóficos. Al escribir estas líneas, mi propósito fue mucho más modesto: intenté transmitir al lector mis recuerdos de una gran personalidad. Pero tengo la triste impresión de un fracaso: no puedo organizar esas memorias para transmitir un retrato convincente, tal vez porque estoy acostumbrado a escribir sobre ideas y no acerca de personas. Como una excusa para este fracaso solo puedo decir que estos son mis recuerdos de Georg Henrik VON WRIGHT, no su biografía ni una evaluación de su filosofía.

Me gustaría concluir este artículo con un puñado de frases tomadas de un trabajo que escribí hace ya más de diez años ${ }^{22}$ :

Durante más de treinta y cinco años nos reunimos frecuentemente con Georg Henrik VON WRIGHT y Elizabeth VON WRIGHT. Cada uno de esos encuentros fue para mí un verdadero «Erlebnis». El aspecto más importante de estas reuniones era la personalidad de Georg Henrik, al que caracterizaría mediante dos palabras alemanas, tomadas de Thomas MANN: «Persönlichkeit» y «mähnschlich». Él fue una extraordinaria «Persönlichkeit», altamente inteligente, increíblemente modesto y profundamente amable. Y él fue profundamente «mähnschlich» en el sentido con que esa palabra es usada con la peculiar entonación de Clawdia CHAUCHAT, la heroína de Zauberberg. La amistad con Georg Henrik y Elizabeth VON WRIGHT forma parte de las experiencias más valiosas de mi vida.

${ }^{21}$ The Tree of Knowledge and other Essays, Lyden-New York-Köln, E. J. Brill, 1993.

22 E. Bulygin, «G. H. von Wright and the Philosophy of Law», in the book quoted in FN 21, 51-58. 